\title{
Interrogation of novel CDK2/9 inhibitor fadraciclib (CYC065) as a potential therapeutic approach for AML
}

Wittawat Chantkran $\mathbb{1}^{1,2}$, Ya-Ching Hsieh ${ }^{1}$, Daniella Zheleva ${ }^{3}$, Sheelagh Frame ${ }^{3}$, Helen Wheadon ${ }^{1}$ and Mhairi Copland (1D)

\begin{abstract}
Over the last 50 years, there has been a steady improvement in the treatment outcome of acute myeloid leukemia (AML). However, median survival in the elderly is still poor due to intolerance to intensive chemotherapy and higher numbers of patients with adverse cytogenetics. Fadraciclib (CYC065), a novel cyclin-dependent kinase (CDK) 2/9 inhibitor, has preclinical efficacy in AML. In AML cell lines, myeloid cell leukemia 1 (MCL-1) was downregulated following treatment with fadraciclib, resulting in a rapid induction of apoptosis. In addition, RNA polymerase II (RNAPII)-driven transcription was suppressed, rendering a global gene suppression. Rapid induction of apoptosis was observed in primary AML cells after treatment with fadraciclib for $6-8 \mathrm{~h}$. Twenty-four hours continuous treatment further increased efficacy of fadraciclib. Although preliminary results showed that AML cell lines harboring KMT2A rearrangement (KMT2A-r) are more sensitive to fadraciclib, we found that the drug can induce apoptosis and decrease MCL-1 expression in primary AML cells, regardless of KMT2A status. Importantly, the diversity of genetic mutations observed in primary AML patient samples was associated with variable response to fadraciclib, confirming the need for patient stratification to enable a more effective and personalized treatment approach. Synergistic activity was demonstrated when fadraciclib was combined with the BCL-2 inhibitor venetoclax, or the conventional chemotherapy agents, cytarabine, or azacitidine, with the combination of fadraciclib and azacitidine having the most favorable therapeutic window. In summary, these results highlight the potential of fadraciclib as a novel therapeutic approach for AML.
\end{abstract}

\section{Introduction}

Acute myeloid leukemia (AML) is one of the most common hematologic malignancies, characterized by clonal, proliferative, and abnormally or poorly differentiated myeloid cells infiltrating the bone marrow, blood or extramedullary tissues ${ }^{1-3}$. Although treatment has progressed, in some AML patients,

\footnotetext{
Correspondence: Mhairi Copland (mhairi.copland@glasgow.ac.uk)

'Paul O'Gorman Leukaemia Research Centre, Institute of Cancer Sciences, College of Medical, Veterinary and Life Sciences, University of Glasgow,

Glasgow, UK

${ }^{2}$ Department of Pathology, Phramongkutklao College of Medicine, Bangkok, Thailand

Full list of author information is available at the end of the article

Edited by Carlo Ganini
}

particularly the elderly ${ }^{1,4}$, outcome is dismal due to the remarkable genetic complexity ${ }^{5,6}$, epigenetic alterations $^{7-9}$, and the dynamics of the disease ${ }^{10,11}$. Treatment-related morbidity/mortality and resistance to chemotherapy are major causes of treatment failure $^{12}$. However, longer life expectancy and possibly a cure is achievable if complete remission (CR) is attained $^{13,14}$. Therefore, there is substantial research to improve and personalize therapies, and lessen treatment toxicity ${ }^{15-18}$.

Currently, several novel agents acting through distinct molecular targets, identified in the pathophysiology of AML, are being investigated alone or combined with conventional chemotherapy, e.g., FLT3 inhibitors

\section{(c) The Author(s) 2021, corrected publication 2021}

\footnotetext{
(c) (i) Open Access This article is licensed under a Creative Commons Attribution 4.0 International License, which permits use, sharing, adaptation, distribution and reproduction in any medium or format, as long as you give appropriate credit to the original author(s) and the source, provide a link to the Creative Commons license, and indicate if changes were made. The images or other third party material in this article are included in the article's Creative Commons license, unless indicated otherwise in a credit line to the material. If material is not included in the article's Creative Commons license and your intended use is not permitted by statutory regulation or exceeds the permitted use, you will need to obtain permission directly from the copyright holder. To view a copy of this license, visit http://creativecommons.org/licenses/by/4.0/.
} 
$\left(\right.$ midostaurin $^{19}$, gilteritinib $^{20}$ ), IDH1/2 inhibitors (ivosidenib $^{21}$, enasidenib ${ }^{22,23}$ ), BCL-2 inhibitor (veneto$\operatorname{clax}^{24,25}$ ), and cyclin-dependent kinase (CDK) inhibitors $\left(\mathrm{CDKi}\right.$; alvocidib ${ }^{26-28}$, fadraciclib $\left.{ }^{29}\right)$, which cause cell cycle arrest and induce apoptosis.

The second generation CDKi fadraciclib is an orally available 2,6,9-trisubstituted purine analog designed to selectively inhibit CDK2 and CDK $9^{29-31}$. Direct inhibition of CDK2 results in cell cycle arrest of abnormally proliferating cells $^{32}$. The blockade of CDK9 halts RNA polymerase II (RNAPII) transcriptional activity consequently inducing apoptosis ${ }^{33}$. Furthermore, CDK9 inhibition downregulates the anti-apoptotic protein myeloid cell leukemia $1(\mathrm{MCL}-1)^{34}$. Fadraciclib has already entered clinical trials in advanced solid tumors (NCT02552953), chronic lymphocytic leukemia (CLL) (NCT03739554) and AML (NCT04017546).

In this study, fadraciclib was assessed for efficacy against AML cell lines and primary AML samples in vitro either as a single agent or in combination with venetoclax (VEN), cytarabine (AraC), or azacitidine (AZA).

\section{Results}

Fadraciclib induces apoptosis of AML cell lines and reduces metabolic activity confirming anti-proliferative effect

IC50 of fadraciclib was established by resazurin reduction assay at 24,48 , and $72 \mathrm{~h}$. The IC50 of fadraciclib was lower at $72 \mathrm{~h}$, compared to 24 and $48 \mathrm{~h}$, showing that longer exposure increased drug potency (Fig. 1A). At $72 \mathrm{~h}$, the IC50 of fadraciclib in OCI-AML3, MOLM-13 and MV4-11 was approximately $0.44 \pm$ $0.01 \mu \mathrm{M}, 0.25 \pm 0.01 \mu \mathrm{M}$ and $0.52 \pm 0.01 \mu \mathrm{M}$, respectively. As compared to the IC50, which is related to antiproliferative activity, higher fadraciclib doses were required to induce apoptosis in $50 \%$ of cells, represented by the percentage of annexin V-positive cells (\%annexinV). $0.75,0.5$, and $1 \mu \mathrm{M}$ of fadraciclib was required to induce apoptosis in 50\% of cells in OCI-AML3, MOLM13 and MV4-11, respectively (Fig. 1B, C). This was confirmed by a significant concentration-dependent increase in the percentage of active caspase-3-positive cells (\%active caspase-3; Fig. 1D). Cell cycle analysis at $72 \mathrm{~h}$ demonstrated a significant increase in the percentage of sub G0 (\%subG0) population at concentrations of $0.75 \mu \mathrm{M}, 0.5 \mu \mathrm{M}$, and $1 \mu \mathrm{M}$ of fadraciclib for OCI-AML3, MOLM-13, and MV4-11, respectively, with no evidence of cell cycle arrest (Fig. 1E). However, at $4 \mathrm{~h}, \mathrm{G} 1$ arrest occurred in OCI-AML3 and MV4-11, but not MOLM13 (Supplementary Fig. S1).

Taken together, fadraciclib potently induces apoptosis, increases the subG0 population, and reduces proliferation as evidenced by reduced metabolic activity in AML cell lines.

\section{Fadraciclib downregulates MCL-1, resulting in rapid induction of apoptosis in AML cell lines}

AML cell lines were treated with $1 \mu \mathrm{M}$ fadraciclib, for 4 and $24 \mathrm{~h}$. An inhibitory effect on downstream targets was demonstrated by immunoblotting (Fig. 2A). Inhibition of CDK2 and CDK9 was evaluated by phosphorylation levels at serine 807/serine 811 of retinoblastoma ( $\mathrm{Rb}$ ) and serine 2 of RNAPII, respectively. Rb was not expressed in OCI-AML3. In MOLM-13, densitometry confirmed a $75 \%$ reduction in $\mathrm{Rb}$ levels by $4 \mathrm{~h}(p<0.0001)$ and greater than $99 \%$ at $24 \mathrm{~h}$, with a corresponding reduction in the phosphorylated form (p-Rb). In MV4-11, only a modest reduction in the levels of $\mathrm{Rb}$ and $\mathrm{p}-\mathrm{Rb}$ at $4 \mathrm{~h}$, with $\mathrm{Rb}$ level returning to baseline and a modest increase in p-Rb observed at $24 \mathrm{~h}$ (Fig. 2A, B). At $4 \mathrm{~h}$, in all cell lines, the level of RNAPII decreased by approximately $60 \%$ with a corresponding decrease in phosphorylation (Fig. 2A, C); with similar results at $24 \mathrm{~h}$.

A significant downregulation of MCL-1 occurred at $4 \mathrm{~h}$ in all cell lines and was maintained or further decreased at 24h (Fig. 2A, D). This induced apoptosis as indicated by the accumulation of cleaved poly (ADP-ribose) polymerase-1 (Fig. 2A, E). The function of CDK1 was not perturbed as observed by an unchanged level of threonine 320 phosphorylated protein phosphatase 1 alpha (Supplementary Fig. S2A, B).

Owing to the impact of fadraciclib on MCL-1 levels, relevant signaling pathways, controlling MCL-1 were investigated. Threonine 180/tyrosine 182 phosphorylated p38 MAPK was markedly increased at $4 \mathrm{~h}$ in MOLM-13 $(p<0.0001)$ and MV4-11 $(p<0.001)$, and at $24 \mathrm{~h}$ in MOLM-13, but not in OCI-AML3 (Supplementary Fig. S2A, C). A decrease in threonine 202/tyrosine 204 phosphorylated Erk1/2 was also observed at 4 and $24 \mathrm{~h}$ (Supplementary Fig. S2A, D). By contrast, serine 473 phosphorylated Akt (Supplementary Fig. S2A, E) and serine 9 phosphorylated GSK3 $\beta$ (Supplementary Fig. S2A, F) were not significantly changed.

These results imply that stress response and apoptosis are potently induced in MOLM-13 and MV4-11 $1^{35-38}$, and the observed downregulation of MCL-1 was unlikely to be via the proteasome-dependent degradation pathway modulated by GSK3 $\beta^{39}$.

\section{Fadraciclib alters cell cycle regulatory gene expression to induce apoptosis}

OCI-AML3, MOLM-13 and MV4-11 were treated with $0.75,0.5$, and $1 \mu \mathrm{M}$ of fadraciclib, respectively, for 4 and $24 \mathrm{~h}$ and the effect on cell cycle regulatory gene expression investigated. The heatmap depicts fold change of gene expression as compared with NDC (Fig. 2F). Several target genes were downregulated following fadraciclib treatment, including; CDK family, transcription factors, key protein phosphatases, CDKI, 

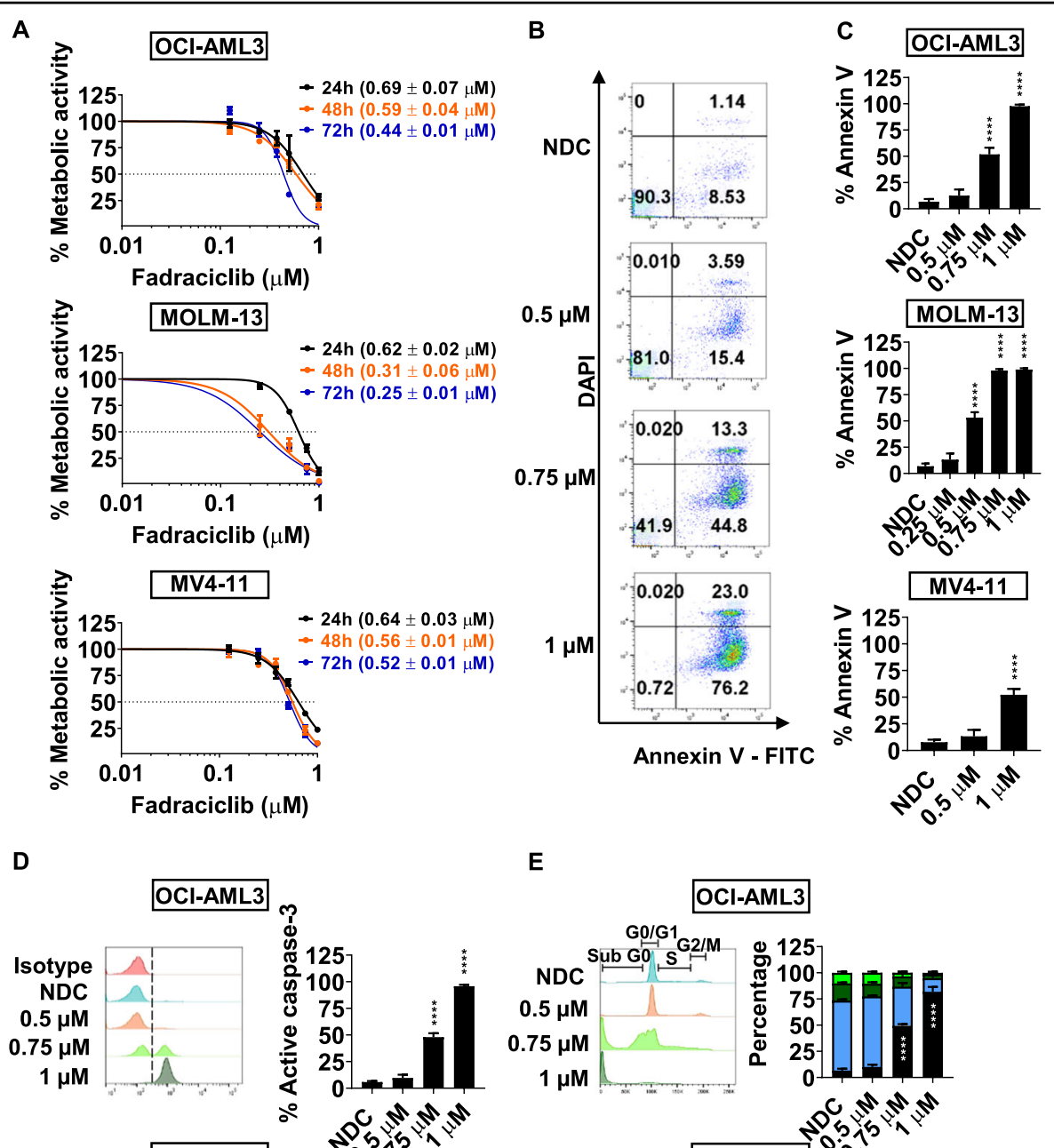

E
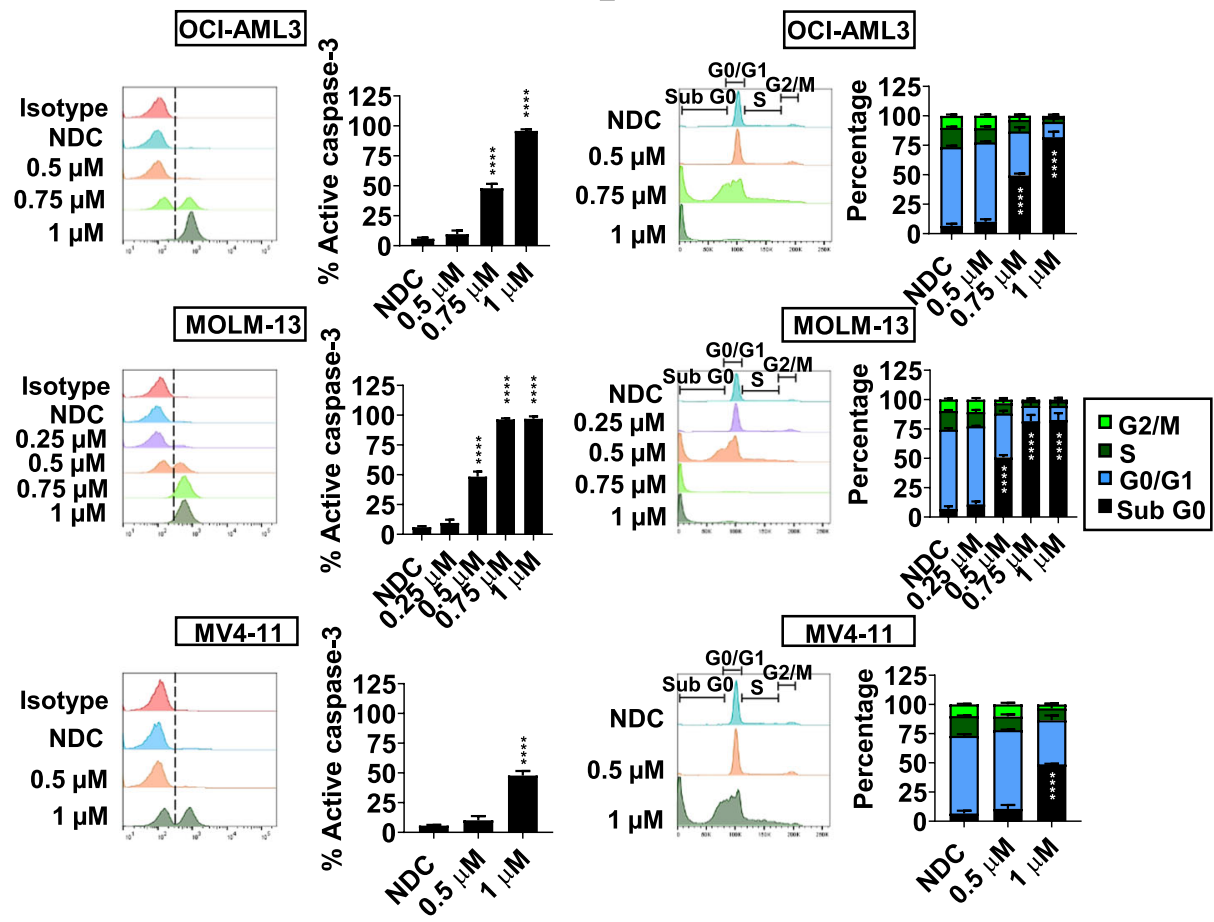

Fig. 1 Fadraciclib reduces metabolic activity and causes apoptosis of AML cell lines. A The IC50 of fadraciclib at 24, 48 and $72 \mathrm{~h}$ in AML cell lines using the rezasurin reduction assay. B Representative flow cytometry plots Annexin V/DAPI apoptosis assay for the OCI-AML3 cell line treated with fadraciclib for $72 \mathrm{~h}$. C Bar charts based on flow cytometry data for the percentage of annexin V-positive cells in AML cell lines treated with fadraciclib for $72 \mathrm{~h}$. D Representative flow cytometry plots and summary bar charts of the percentage of active caspase-3-positive cells in AML cell lines treated with fadraciclib for $72 \mathrm{~h}$. E Representative flow cytometry plots and summary bar charts of cell cycle phase using PI staining of AML cell lines treated with fadraciclib for $72 \mathrm{~h} . n=3$.Graphs depict mean \pm SD $\left({ }^{* * *} p<0.0001\right)$. Data were compared using ANOVA. 


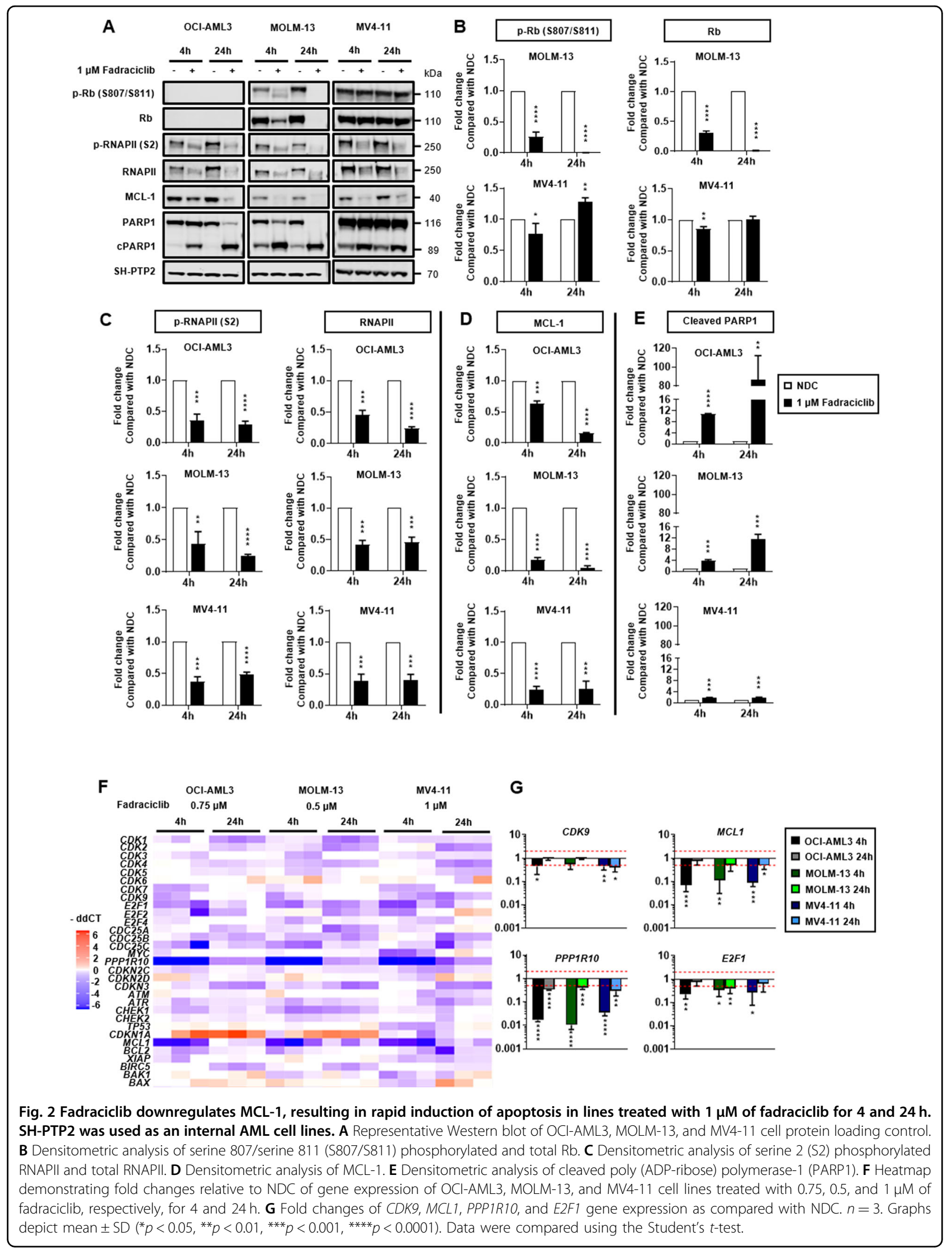


DNA damage response regulators, anti-apoptotic related, and pro-apoptotic related genes (Supplementary Figs. S3-S5).

In particular, at $4 \mathrm{~h}$, the expression of CDKs, especially $C D K 9$, the transcription factor $E 2 F 1$, and $M C L 1$, were suppressed. PPP1R10 was also significantly downregulated (Fig. 2F, G). Effects on gene expression were less marked at $24 \mathrm{~h}$. Conversely, the DNA damage response regulator gene $C D K N 1 A$, encoding $\mathrm{p} 21^{\mathrm{Cip} 1}$, was upregulated at $24 \mathrm{~h}$, indicating the intrinsic apoptosis pathway ${ }^{40}$ was activated following fadraciclib treatment (Supplementary Fig. S4B).

\section{Fadraciclib induces apoptosis and decreases MCL-1 expression in primary AML cells, regardless of KMT2A-PTD status}

Published results indicate AML cell lines bearing $K M T 2 A$ rearrangements $(K M T 2 A-r)$ are sensitive to fadraciclib $^{29}$. Five primary AML samples harboring KMT2A-partial tandem duplication (PTD) and five primary AML samples with KMT2A wild-type (WT) were tested with fadraciclib as a single agent (Table. 1). All samples were treated with 0.5 and $1 \mu \mathrm{M}$ fadraciclib for $24 \mathrm{~h}$, then the drug was washed out and cells were cultured in fresh media for up to $72 \mathrm{~h}$.

At 24,48 , and $72 \mathrm{~h}$ time points, fadraciclib treatment resulted in an increase in apoptosis in both KMT2APTD and KMT2A WT patient samples (Fig. 3A, B). The proportion of apoptotic cells significantly increased over time. Specifically, at $72 \mathrm{~h}$, a concentrationdependent maximal increase in \%annexinV was observed in KMT2A-PTD patient samples following treatment with fadraciclib at $0.5 \mu \mathrm{M}(20.5 \pm 9.6 \%, p=$ $0.6051)$ and $1 \mu \mathrm{M}(79.5 \pm 12.9 \%, p<0.0001)$, and in $K M T 2 A$ WT patient samples following treatment with fadraciclib at $0.5 \mu \mathrm{M}(33 \pm 14.6 \%, p=0.624)$ and $1 \mu \mathrm{M}$ $(74 \pm 26 \%, p<0.0001)$ compared with NDC (Fig. 3A). Responses were similar in KMT2A-PTD and KMT2A WT patient samples.

A concentration-dependent increase in \%active caspase3 was observed in both KMT2A-PTD and KMT2A WT patient samples following fadraciclib treatment (Fig. 3B). However, due to genetic heterogeneity of AML samples (Table. 1), this difference did not reach statistical significance.

Treatment with fadraciclib resulted in a time- and concentration-dependent decrease in MCL-1 expression in both KMT2A-PTD and WT AML samples. Specifically, at $72 \mathrm{~h}$, a decrease in MCL-1 in KMT2APTD patient samples at $0.5 \mu \mathrm{M}(42.8 \pm 20.3 \%, p=$ $0.9997)$ and $1 \mu \mathrm{M}(5.4 \pm 3.2 \%, p=0.1852)$, and in $K M T 2 A$ WT patient samples at $0.5 \mu \mathrm{M}(31.2 \pm 21.2 \%, p$ $=0.9463)$ and $1 \mu \mathrm{M}(4 \pm 1 \%, p=0.1913)$ following fadraciclib treatment compared with NDC (Fig. 3C).
Pulsed dosing with fadraciclib in primary AML cells results in apoptosis and reduces cell viability, but is less effective than continuous dosing

To determine if pulsed dosing achieved an optimal response, primary AML cells were treated for up to $8 \mathrm{~h}$ with fadraciclib at a concentration of $1 \mu \mathrm{M}$. This resulted in a significant increase in \%annexinV at both 6 and $8 \mathrm{~h}$ $(p=0.023$ and $p=0.0411$, respectively) and \%active caspase-3 at $6 \mathrm{~h}(p=0.0351)$ as compared with NDC (Supplementary Fig. S6A, B). As fadraciclib exerts a rapid effect, we investigated whether a 6-h pulse was as effective as 24-h continuous treatment at inducing apoptosis. Fadraciclib showed a higher \%active caspase-3 after continuous treatment compared to the pulsed treatment (Supplementary Fig. S6C). MCL-1 levels were significantly reduced after 24-h continuous treatment compared to both the NDC and the 6-h pulsed treatment $(p=0.0167$ and $p=0.0304$, respectively; Supplementary Fig. S6D).

\section{Increased apoptosis of primary human AML samples in combination studies of fadraciclib + VEN, fadraciclib + AraC, and fadraciclib + AZA}

For combination studies, six primary AML samples were selected based on the presence of the most common favorable (e.g., NPM1) and unfavorable mutations (e.g., FLT3-internal tandem duplication (ITD) and KMT2APTD), in order to assess the efficacy of fadraciclib and its synergistic activity when combined with VEN, AraC, or AZA, towards these mutations.

Drug combination studies exploit opportunities for reduced drug resistance, decreased toxicity, and efficacy improvement ${ }^{41}$. Based on phase 1 clinical trial data, the maximum clinically achievable concentration of fadraciclib is $6-7 \mu \mathrm{M}$ when administered as a single agent ${ }^{42}$. In this study, much lower fadraciclib concentrations of $0.25 \mu \mathrm{M}$ (FAD1) and $0.5 \mu \mathrm{M}$ (FAD2) in combination with VEN, AraC or AZA were assessed for efficacy against primary AML cells. The concentrations selected were based on previous studies: $0.025 \mu \mathrm{M}$ (VEN1) and $0.5 \mu \mathrm{M}^{43}$ (VEN2); $0.01 \mu \mathrm{M}$ (AraC1) and $0.1 \mu \mathrm{M}^{44}$ (AraC2); $0.5 \mu \mathrm{M}^{45}$ (AZA1) and $2 \mu \mathrm{M}^{46}$ (AZA2), aiming to optimize results for both sensitive and more therapy-resistant patient samples. All drug concentrations were within clinically achievable concentrations $\left(1 \mu \mathrm{M} \mathrm{VEN}^{47}, 1 \mu \mathrm{M} \mathrm{AraC}^{48}\right.$, and $\left.3-11 \mu \mathrm{M} \mathrm{AZA}^{49}\right)$. Apoptosis, active caspase-3, cell cycle analyses, and proliferation assays were performed at $72 \mathrm{~h}$.

In primary AML samples, \%annexinV and \%active caspase-3 (Supplementary Figs. S7A-C and S8A-C, respectively) were not significantly different at low concentrations, with any drug combination pair tested. At high concentrations, a modest increase in \%annexinV was observed; $82.2 \pm 16.9 \%$ in FAD2 + VEN2 combination (as compared with $55.7 \pm 14 \%$ in FAD2, $p=0.1059$; and 


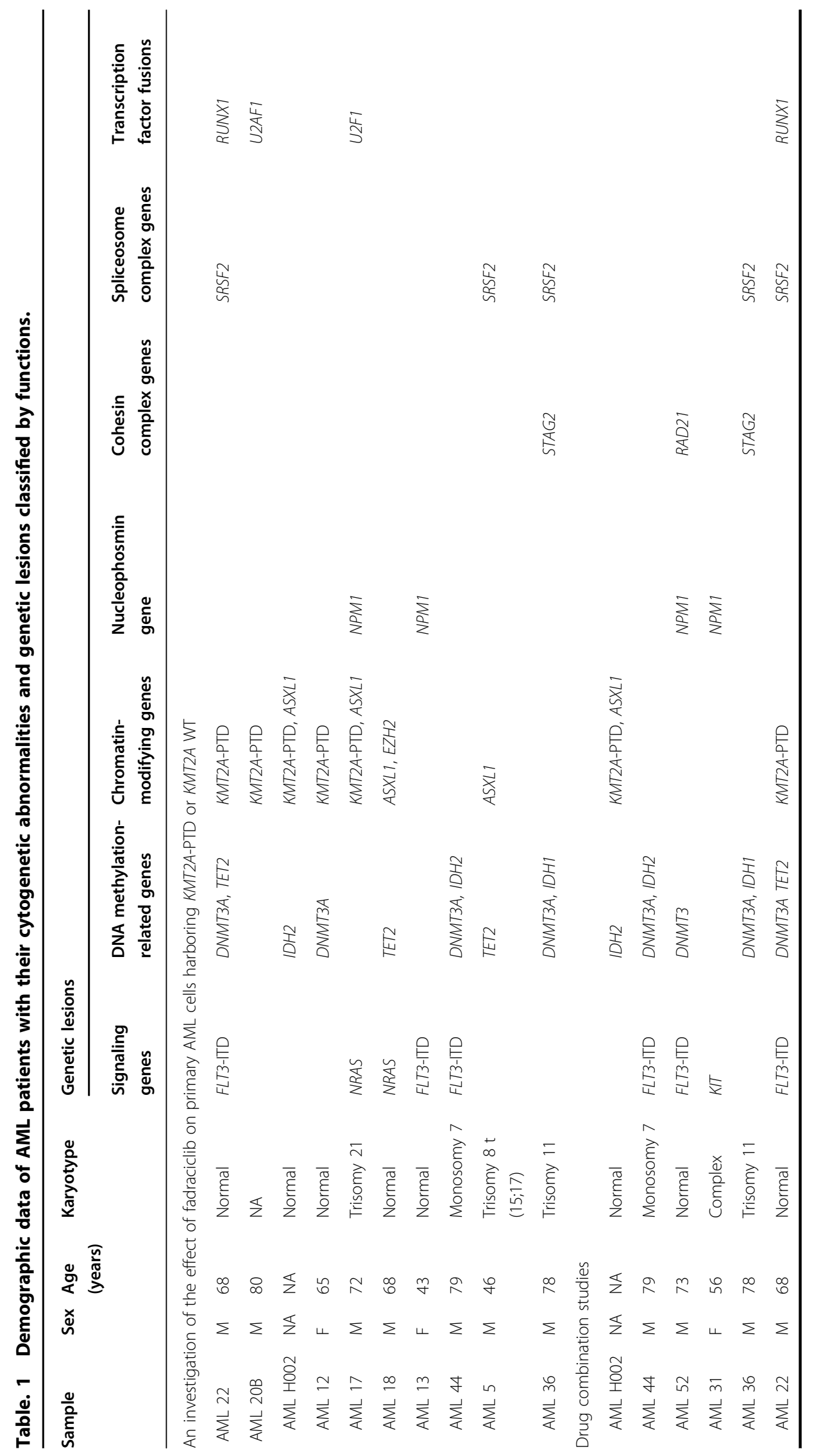




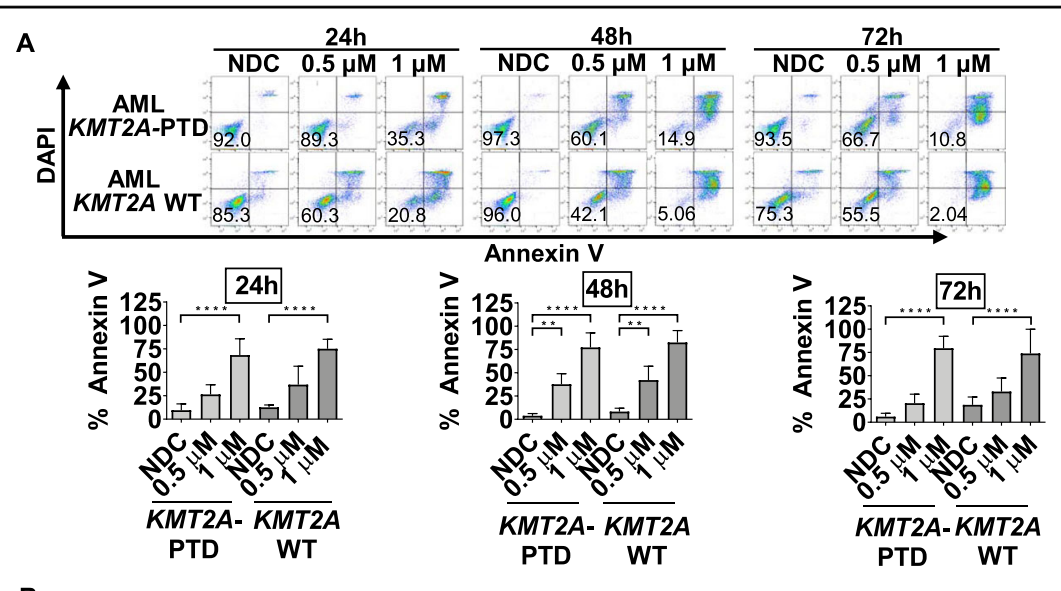

B
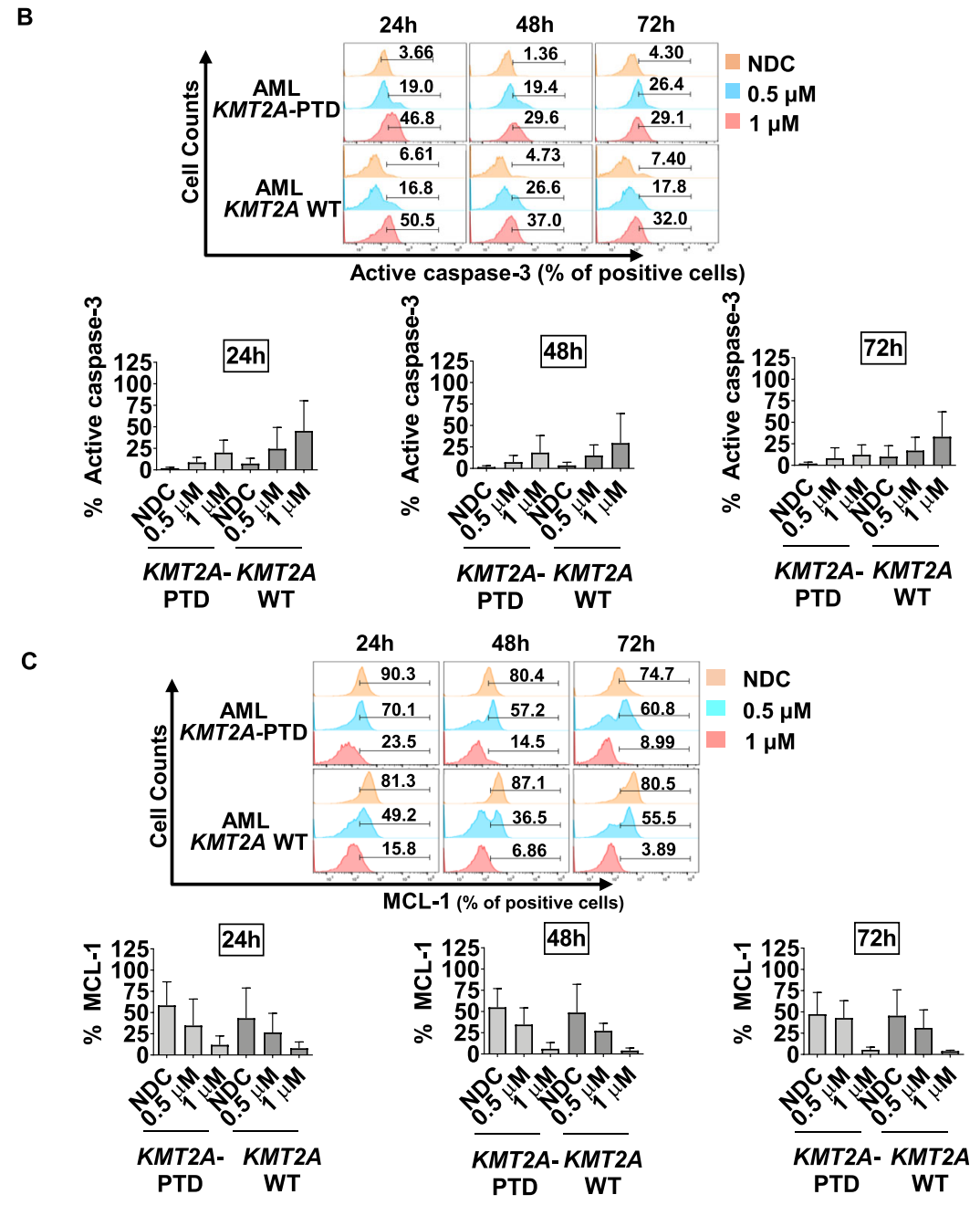

Fig. 3 Fadraciclib induces apoptosis and decreased MCL-1 expression in primary AML cells, regardless of KMT2A-PTD status. All samples were treated with $0.5 \mu \mathrm{M}$ or $1 \mu \mathrm{M}$ of fadraciclib for $24 \mathrm{~h}$, then the drug was washed out and cells were cultured in fresh media for up to $72 \mathrm{~h}$. A Representative flow cytometry plots and summary bar charts of the percentage of annexin V-positive cells. $n=4-5$. B Representative flow cytometry plots and summary bar charts of the percentage of active caspase-3-positive cells. $n=4-5$. C Representative flow cytometry plots and summary bar charts of the percentage of the MCL-1 level. $n=3$. Graphs depict mean \pm SD $\left({ }^{* *} p<0.01,{ }^{* * * *} p<0.0001\right)$. Data were compared using ANOVA. 


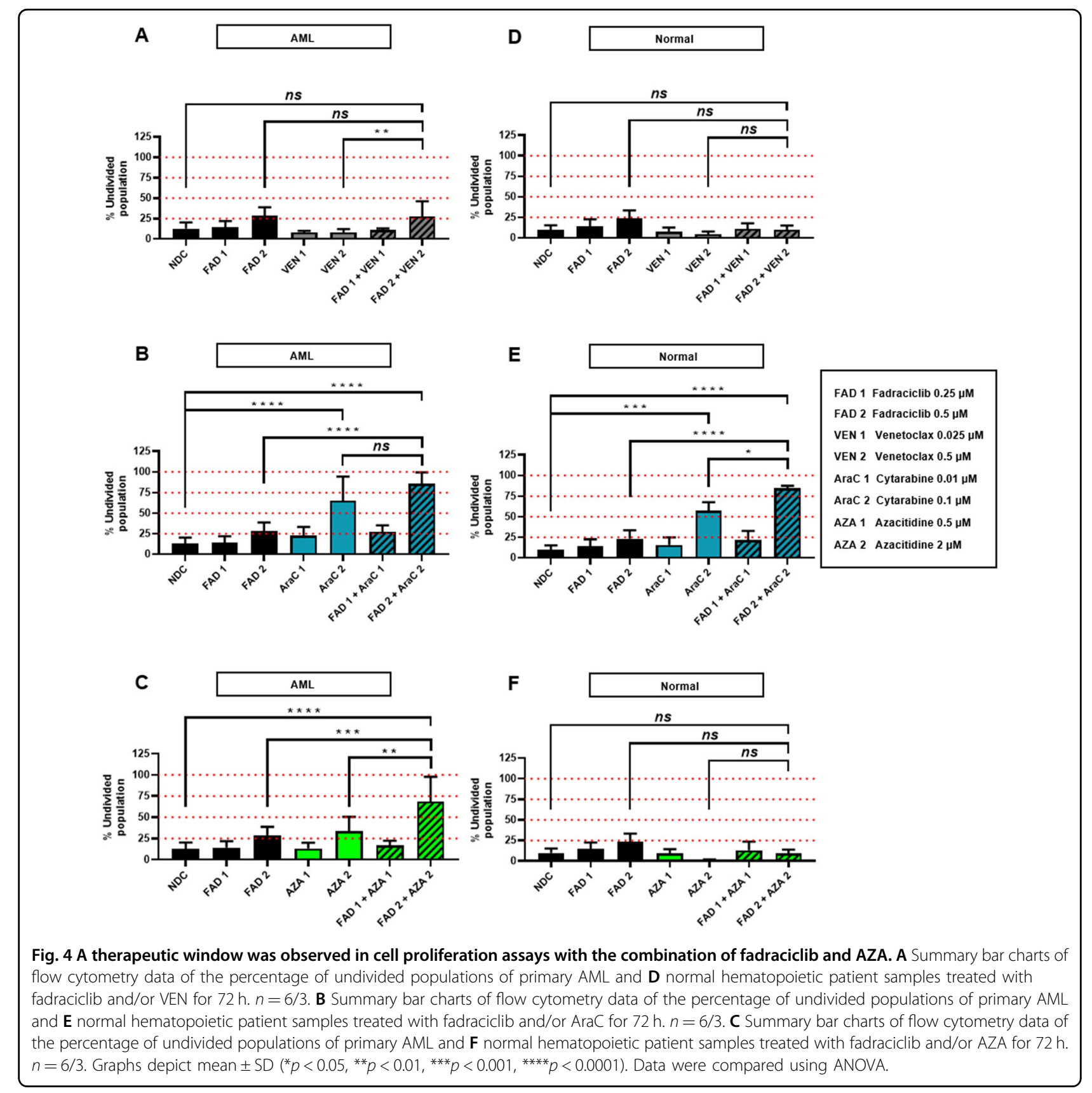

$64.6 \pm 21.4 \%$ in VEN2 alone, $p=0.5166$ ) (Supplementary Fig. S7A), $81.6 \pm 13.4 \%$ in FAD2 + AraC2 combination (as compared with $55.7 \pm 14 \%$ in FAD2, $p=0.1077$; and $69.5 \pm 22.5 \%$ in AraC2 alone, $p=0.8117$ ) (Supplementary Fig. S7B), and $87.7 \pm 12.3 \%$ in FAD2 + AZA2 combination (as compared with $55.7 \pm 14 \%$ in FAD2, $p=0.0109$; and $70.7 \pm 22.8 \%$ in AZA2 alone, $p=0.437$ ) (Supplementary Fig. S7C). Consistently, a modest increase in \%active caspase-3 was observed at high concentrations; $84.3 \pm$ $11.5 \%$ in FAD2 + VEN2 combination (as compared with $55.6 \pm 14 \%$ in FAD2, $p=0.0217$; and $65.2 \pm 17.9 \%$ in VEN2 alone, $p=0.267$ ) (Supplementary Fig. S8A), $86.8 \pm$
$5.9 \%$ in $\mathrm{FAD} 2+\mathrm{AraC} 2$ combination (as compared with $55.6 \pm 14 \%$ in FAD2, $p=0.0143$; and $69.3 \pm 19.5 \%$ in AraC2 alone, $p=0.4081$ ) (Supplementary Fig. S8B), and $90.7 \pm 4 \%$ in FAD2 + AZA2 combination (as compared with $55.6 \pm 14 \%$ in FAD2, $p=0.0006$; and $74.4 \pm 17.3 \%$ in AZA2 alone, $p=0.3197$ ) (Supplementary Fig. S8C).

Cell cycle analysis revealed \%subG0, but no other cell cycle phase, marginally increased in agreement with the increase in \%annexinV and \%active caspase- 3 observed in FAD2 + VEN2, FAD2 + AraC2 and FAD2 + AZA2 combination (Supplementary Fig. S9A-C) as compared with each single drug treatment. Overall results indicate the 
more complex the molecular genetic lesions or complexity of karyotype, the less efficacious the combination therapy was (Supplementary Table S4).

\section{Fadraciclib alone and low-dose combination therapies have limited cytotoxicity on normal hematopoietic cells}

Fadraciclib alone and in combination with VEN, AraC and AZA was assessed in normal CD34 + hematopoietic samples to determine toxicity. Neither fadraciclib alone nor lower concentration drug combinations exerted a significant increase in \%annexinV, \%active caspase-3 or \% subG0 (Supplementary Figs. S7D-F, S8D-F, and S9D-F, respectively) as compared to NDC. However, as expected, an increase in \%annexinV was observed at high concentrations with a corresponding increase in \%active caspase-3 (Supplementary Figs. S7D-F and S8D-F, respectively).

\section{Fadraciclib + AZA combination therapy has a therapeutic} window in in vitro cell proliferation assays

Finally, cell proliferation assays using CellTrace Violet staining were performed. Representative histograms for AML and normal CD34 + samples are shown (Supplementary Fig. S10). When considering the combination therapies, none of the low concentration drug combinations resulted in a significant anti-proliferative effect (increased percentage of undivided population, \%Undivided \%undivided) compared to either NDC or single drug therapies in either AML or normal CD34 + samples (Fig. 4A-F).

However, differences occurred with the high concentration drug combinations. Specifically, FAD2 + VEN2, had significant anti-proliferative effects when compared to VEN2, but might be explained by the effect of FAD2 (Fig. 4A). This combination had no antiproliferative effect on normal CD34 + cells (Fig. 4D). Although FAD2 + AraC2, has significantly increased anti-proliferative effect as compared to FAD2 in both AML and normal CD34 + samples, this is fully accounted for by the known cytotoxic effects of AraC (Fig. 4B, E). For FAD2 + AZA2, the anti-proliferative effect in AML samples was significantly increased compared to both FAD2 and AZA2 (\%Undivided \% undivided of $68.7 \pm 29.1 \%$ for FAD2 + AZA2 compared with $28.5 \pm 10.2 \%$ in FAD2, $p=0.0004$; and $33.5 \pm 17 \%$ in AZA2, $p=0.0024$ ), as well as NDC (Fig. 4C), indicating synergism of this combination with a combination index (CI) of 0.75 . No anti-proliferative effect was demonstrated for this combination on normal CD34+ cells (Fig. 4F).

These findings indicate a therapeutic window and provide evidence for further exploration of combination treatment with fadraciclib and AZA as a promising treatment approach.

\section{Discussion}

Our study evaluated the therapeutic potential of fadraciclib in AML. AML cell lines harboring various genetic lesions were tested, these include OCI-AML3 (carries NPM1 and DNMT3A ${ }^{\mathrm{R} 882 \mathrm{C}}$ mutations), MOLM-13 (carries FLT3-ITD and KMT2A-MLLT3), and MV4-11 (carries FLT3-ITD and KMT2A-AFF1), aiming to encompass stratification of major risk profiles according to the European LeukemiaNet recommendations ${ }^{50}$. Results indicate MV4-11, harboring adverse prognosis molecular abnormalities, was less sensitive with the highest IC50 (Fig. 1A) and a higher dose of fadraciclib required to induce apoptosis (Fig. 1C-E).

Inhibition of CDK2 and CDK9 was evaluated by phosphorylation of $\mathrm{Rb}^{51,52}$ and RNAPII ${ }^{53,54}$. We found the effects of fadraciclib on $\mathrm{Rb}$ were cell line specific (Fig. 2A, $B$ ), whereas, the endogenous level of RNAPII was decreased in all cell lines (Fig. 2A, C). A transcriptional downregulation of RNAPII is the most plausible explanation for the global gene suppression observed (Fig. 2F and Supplementary Figs. S3-S5). This is consistent with a previous study stating that a downregulation of POLR $2 \mathrm{~A}$ encoding the major subunit of RNAPII was observed in leukemic blasts following treatment with alvocidib, which potently inhibits $C D K 9^{55}$. A significant decrease in short half-life MCL-1 at gene (Fig. 2G) and protein (Fig. 2A, D) levels highlights a potential target inhibited by fadraciclib.

A rapid decrease in MCL-1 level perturbs a balance in the BCL-2 family, activating the intrinsic apoptosis pathway (Fig. 5B). AML cells are more dependent on MCL-1 than normal hematopoietic cells ${ }^{56}$, highlighting the potential of fadraciclib as a therapeutic approach for AML. Downregulation of E2F1 (Fig. 2G) may explain the G1 cell cycle arrest seen in OCI-AML3 and MV4-11 following fadraciclib treatment (Supplementary Fig. S1). Interestingly, Erk1/2 were suppressed in all cell lines tested (Supplementary Fig. S2A, D), which potentially promotes a reduction in MCL-1 stability and half-life ${ }^{57}$. In various cancer cell lines, siRNA-mediated PPP1R10 knockdown results in tumor suppressor PTEN release from nuclear sequestration ${ }^{58}$. Hence, the significant decrease in PPP1R10 gene expression observed (Fig. 2G) potentially promotes an induction of apoptosis. Using leukemic blasts from adult patients with refractory AML in a phase 1 clinical trial (NCT00470197), treatment with the pan-CDKi alvocidib, resulted in a downregulation of various genes ${ }^{55}$. Among these, downregulation of POLR2A, encoding the major subunit of RNAPII, and $E 2 F 1$ was seen. In addition, a decrease in phosphorylation of RNAPII and expression of MCL-1 was observed in leukemia blasts of some patients treated with alvocidib who achieved a $\mathrm{CR}^{59}$. Favorably, we found that the function of CDK1, which plays an important role in mitosis $^{60,61}$, was not perturbed, suggesting better immune 


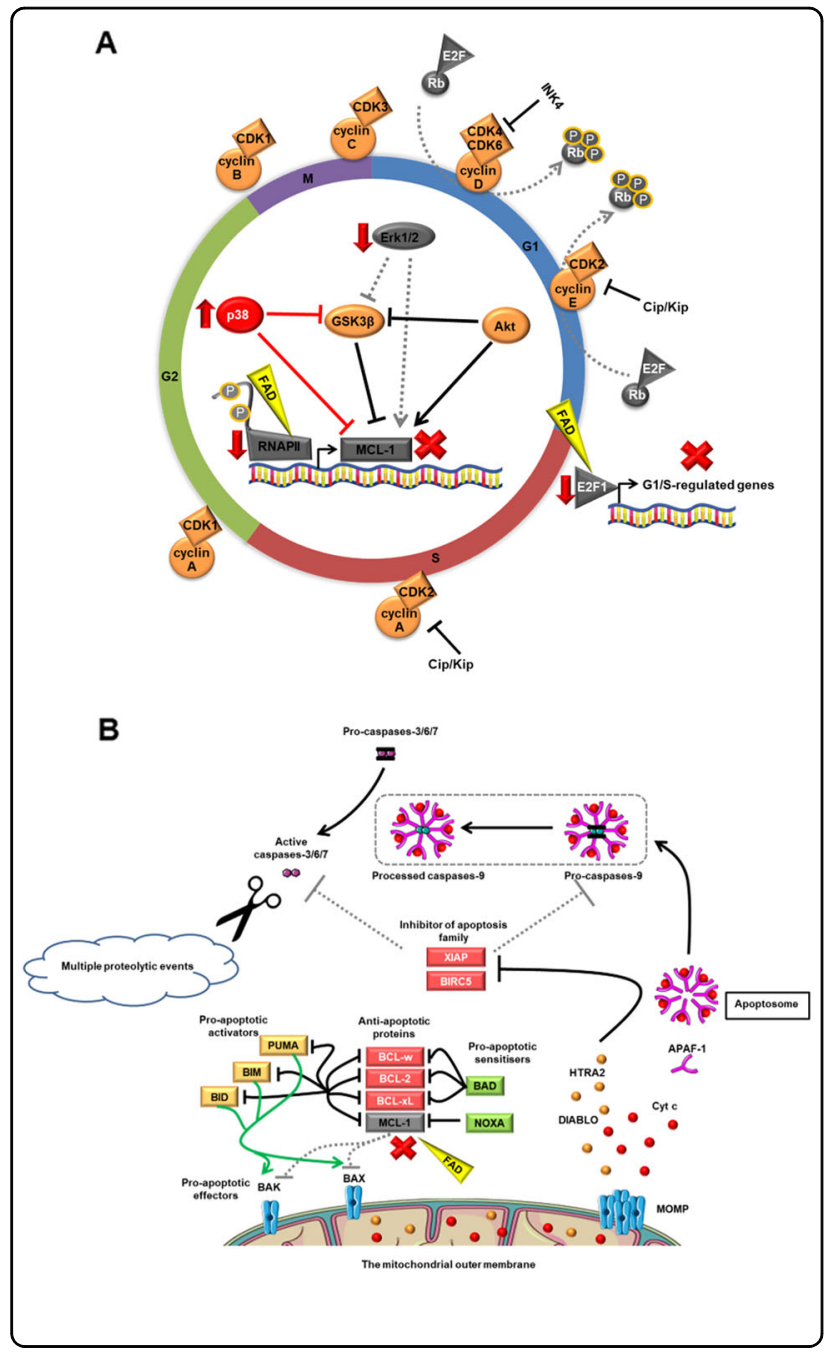

function with less impact on normal hematopoietic cells following fadraciclib treatment.

Recently, a phase 1 clinical trial of fadraciclib in solid tumor patients was completed (NCT02552953) ${ }^{42}$. Doselimiting toxicities were reversible and a biologically effective dose of $192 \mathrm{mg} / \mathrm{m}^{2} /$ day, which corresponds to the drug concentration of $6-7 \mu \mathrm{M}$ in vitro was established $^{42}$.

Over the past few years, many targeted therapies for patients with AML have emerged with efficacy dependent on molecular subtype of $\mathrm{AML}^{62}$. This highlights the important of assessing the efficacy of fadraciclib on specific AML subtypes based on its mechanism of action of inhibiting CDK9. As part of the Super Elongation Complex (SEC), CDK9 is important for transcriptional elongation, in particular in KMT2A-r AML ${ }^{63}$, it is therefore rational to evaluate the efficacy of fadraciclib in this AML subtype. Preliminary results indicate KMT2A-r AML cell lines are more sensitive to fadraciclib ${ }^{29}$. Patient samples, however, display a high diversity of genetic mutations
Fig. 5 A schematic diagram of the effects of fadraciclib at $4 \mathrm{~h}$. A Fadraciclib downregulates $E 2 F 1$, resulting in cell cycle arrest at G1 phase. Downregulation of RNAPII is the most plausible explanation for the global gene suppression observed. A rapid decrease in short halflife $\mathrm{MCL}-1$ level perturbs a balance in the $\mathrm{BCL}-2$ family. A decrease in the Erk1/2 activity observed in all cell lines tested potentially promotes a reduction in MCL-1 stability and half-life. p38 MAPK is markedly increased in MOLM-13 cell line only, implying that the stress response and apoptosis are highly induced. B Fadraciclib downregulates MCL-1, which activates the intrinsic apoptosis pathway, followed by cell death. Indeed, a rapid decrease in $\mathrm{MCL}-1$ level perturbs a balance in the $\mathrm{BCL}-2$ family, which leads directly to activator $\mathrm{BH} 3$-only proteinsbinding BAK and BAX, resulting in their homo-oligomerisation and MOMP. Following this, pro-apoptotic proteins within the mitochondrial intermembrane space, for example, Cyt c, DIABLO and HTRA2 are released. Cyt c binds to APAF-1 to form the apoptosome. Once formed, the apoptosome can then recruit and activate the inactive pro-caspase-9. Following this, caspases-3, 6, and 7 are activated and multiple proteolytic events occur. FAD, Fadraciclib; Cip/ Kip, cyclin-dependent kinase interacting protein/kinase inhibitory protein; INK4, inhibitors of cyclin-dependent kinase 4; G1, Gap 1 phase; S, Synthesis phase; G2, Gap 2 phase; M, Mitotic phase; BCL-2, B-cell lymphoma 2; BCL-XL, B-cell lymphoma-extra large; BCL-w, B-cell lymphoma 2-like protein 2; BAD, B-cell lymphoma 2-associated death promoter; NOXA, phorbol-12-myristate-13-acetate-induced protein 1; BIM, B-cell lymphoma 2-like protein 11; BID, BH3 interacting-domain death agonist; PUMA, p53 upregulated modulator of apoptosis; BAK, B-cell lymphoma 2 homologous antagonist/killer; BAX, B-cell lymphoma 2-associated $X$ protein; MOMP, mitochondrial outer membrane permeabilisation; Cyt c, cytochrome c; DIABLO, direct inhibitor of apoptosis proteins-binding protein with low pl; HTRA2, high-temperature requirement A serine peptidase 2; APAF-1, apoptotic protease activating factor-1; BIRC5, baculoviral inhibitor of apoptosis repeat-containing 5; XIAP, X-linked inhibitor of apoptosis protein.

(Table. 1). We therefore observed a more variable fadraciclib response with $K M T 2 A$-PTD status not being a predictor for better treatment response. This is consistent with previous results showing that the CDK9-specific inhibitor atuveciclib inhibits the proliferation of seven AML cell lines, regardless of $K M T 2 A-r$ status ${ }^{64}$. In addition, atuveciclib displayed potent in vitro activity in $80 \%$ of AML patient samples harboring KMT2A WT, including those with mutant NPM1 or FLT3-ITD.

Considering the combination studies, primary AML samples were selected based on the most common favorable (e.g., NPM1) and unfavorable mutations (e.g., FLT3-ITD and KMT2A-PTD). Initially, we hypothesized that the treatment response would be relevant to the risk profiles of the individual genetic lesions in primary AML samples. However, we found that complexity of the molecular genetic lesions and karyotypes have more influence. The more complex the molecular landscape, the less efficacious the combination therapy (Supplementary Table S4). The treatment response variability associated with the high diversity of genetic mutations 
observed reiterates the results of previous experiment showing the variable fadraciclib response, regardless of KMT2A-PTD status. Taken together, our results highlight the importance of genetic and molecular profiling prior to initiating therapy, to identify personalized therapeutic combinations most likely to benefit individual patient. Regarding the 2017 ELN risk stratification ${ }^{50}$, next generation sequencing (NGS), exome sequencing and genome wide assays ${ }^{65}$, are being developed to replace single gene assays. This will facilitate better prognostication and more personalized therapy in the future ${ }^{66}$. A short delay in commencing therapy in newly diagnosed AML had no effect on outcome after accounting for other prognostic covariates ${ }^{67}$, providing a potential window to perform NGS.

As a global effect, an increase in cell death in primary human AML cells was shown for apoptosis, active caspase- 3 assays, and cell cycle analysis when fadraciclib was combined with VEN, AraC, or AZA. In primary AML cells, combining the MCL-1 inhibitor S63845 with VEN results in greater efficacy than either inhibitor alone, with a more potent activity against leukemic rather than normal hematopoietic progenitors ${ }^{68}$. In an AML patientderived xenograft mouse model bearing FLT3-ITD, the CDK9 inhibitor A-1592668 combined with VEN provided a significant survival advantage over single treatments ${ }^{69}$. In a lymph node mimicking microenvironment, fadraciclib in combination with VEN for $24 \mathrm{~h}$ efficiently induced apoptosis of primary CLL cells ${ }^{70}$, a disease where MCL-1 plays a role in disease progression and fludarabine resistance ${ }^{71}$. Ongoing phase 1 clinical trials are evaluating the safety and tolerability of fadraciclib in combination with VEN in patients with relapsed/refractory AML or myelodysplastic syndromes (MDS) (NCT04017546); and relapsed/refractory CLL (NCT03739554).

When considering combining CDK9 inhibitors with AraC, a recent phase 2 study (NCT01349972), demonstrated a significantly higher efficacy of FLAM (alvocidib, cytarabine plus mitoxantrone) as compared with the standard “ $7+3$ " regimen in terms of a CR $\operatorname{rate}^{26,28}$. However, no increase in overall or event-free survival rates were observed. Potentially, this may be due to the higher toxicity of the combination regimen based on our preliminary data showing the impact of the fadraciclib + AraC on normal hematopoietic cells.

In preliminary experiments, pre-treatment with alvocidib reduced the IC50 of AZA in MV4-11 cell line ${ }^{72}$. In the same manner seen in fadraciclib + AZA combination presented here, alvocidib + AZA also increased \%active caspase-3 as compared with single treatments. In a MOLM-13 xenograft model, the combination showed greater tumor growth inhibition compared to single agent treatments. Our results, demonstrated a potential therapeutic window with the FAD2 + AZA2 combination, as measured by \%Undivided \%undivided of AML cells in cell proliferation assay (Fig. 4C). These interesting results identify an attractive therapeutic strategy warranting consideration of clinical development in AML.

In summary, our studies indicate the preclinical efficacy of the CDK2/9 inhibitor fadraciclib as a single agent or in conjunction with frontline AML chemotherapeutics, highlighting its potential as a novel therapeutic approach. Our work implicates the importance of molecular genetic profiling as a critical step prior to initiating therapy for AML, highlighting the need for a more personalized medicine approach to improve outcomes for patients.

\section{Materials and methods}

\section{Cell lines and primary AML samples cell culture}

OCI-AML3, MOLM-13, and MV4-11 (DSMZ) were cultured at $37^{\circ} \mathrm{C}$ in a $5 \% \mathrm{CO}_{2}$ atmosphere in RPMI 1640 media (Sigma-Aldrich) with $20 \%$ or $10 \%$ fetal bovine serum (Gibco), supplemented with $1 \mathrm{mM}$ glutamine and $1 \%$ penicillin-streptomycin (Invitrogen).

Healthy donor and diagnostic primary AML samples were taken in accordance with the Declaration of Helsinki, and Ethics Committee approval (15/WS/0077). Demographic data, cytogenetic abnormalities and genetic lesions of AML patients are shown in Table. 1. Primary samples were thawed and cultured overnight in serumfree medium II (Stem Cell Technologies) supplemented with hIL-3, hIL-6, hSCF, and hFLT3L (all at $10 \mathrm{ng} / \mathrm{mL}$ ) (Stem Cell Technologies).

\section{Inhibitors}

Ten micromolar stock solutions of fadraciclib (Cyclacel), AZA (Stratech), and VEN (Stratech) were prepared in DMSO. $10 \mathrm{mM}$ AraC (Sigma-Aldrich) was prepared in water. All stocks were stored at $-20^{\circ} \mathrm{C}$ and dilutions freshly prepared in cell culture media.

\section{Rezasurin reduction assay}

Half-maximal inhibitory concentration (IC50) of AML cell lines treated with fadraciclib for up to $72 \mathrm{~h}$ was established using 7-Hydroxy-3H-phenoxazin-3-one-10oxide sodium salt resazurin assay (Sigma-Aldrich) according to manufacturer's instructions. IC50 were calculated using GraphPad Prism 8 software (GraphPad Software).

\section{Gene expression analysis}

RNA was extracted using RNA Easy Micro kits (Qiagen) and converted to cDNA using high-capacity cDNA reverse transcription kit (Applied Biosystems). Quantitative reverse transcription polymerase chain reaction (qRT-PCR) was performed using Fluidigm Biomark technology and data were collected as per manufacturer's instructions. Data was analyzed using the $2^{-\Delta \Delta C T}$ 
compared to no drug control (NDC) as previously described $^{73}$. Internal sample control was ensured by subtracting the average of six housekeeping genes $A T P 5 F 1 B, B 2 M, C Y C 1, R N F 20, T Y W 1$, and UBE2D2 from the $\mathrm{Ct}$ value of each gene of interest. Mean and standard deviation of fold change in expression were calculated. For Primer sequences see Supplementary Table S1.

\section{Protein extraction and quantification}

In all, $1-5 \times 10^{6}$ cells were harvested and washed twice in ice-cold PBS and pelleted at $300 \times g$ for $5 \mathrm{~min}$. Then, the pellets were lyzed in ice-cold solubilization buffer containing phosphatase and protease inhibitors at a concentration of $1 \times 10^{6}$ cells per $10 \mu$ l solubilization buffer (50 mM Tris- $\mathrm{HCl}$ pH 7.5, $150 \mathrm{mM} \mathrm{NaCl}, 1 \%$ Nonidet P40, 10\% Glycerol, cOmplet ULTRA 1 tablet and PhosSTOP phosphatase inhibitor 1 tablet per $10 \mathrm{~mL}$ of solubilization buffer). This suspension was left on ice and vortexed every $15 \mathrm{~min}$ for $1 \mathrm{~h}$, then centrifuged at $14,000 \times g$ for $10 \mathrm{~min}$ to pellet all cell debris. The supernatant was transferred to a fresh eppendorf and stored at $-80^{\circ} \mathrm{C}$ until required.

Protein concentration was quantified using the Quick Start Bradford Dye reagent and compared to a standard curve prepared using Quick StartTM BSA protein. The colorimetric protein assay was measured by visual absorbance on the Spectramax M5 plate reader at $595 \mathrm{~nm}$ at using SoftMax Pro software.

\section{Protein analysis}

Cell lysates were prepared and $30 \mu \mathrm{g}$ of protein was resolved. Western blotting was performed using the NuPAGE electrophoresis system (Invitrogen) as per manufacturer's instructions, and protein detection via Odyssey Fc Imaging System. Densitometry was performed using Image Studio Lite version 5.2. Antibodies and experimental conditions are outlined in Supplementary Table S2.

\section{Flow cytometry}

For proliferation analysis, primary AML and normal CD34+ cells were stained with CellTrace Violet (Thermo Fisher Scientific) as per manufacturer's protocol. For establishing a maximum point of fluorescence staining, cells were cultured with Colcemid $(100 \mathrm{ng} / \mathrm{mL}$, SigmaAldrich) to determine non-dividing cells. All primary cells were treated with fadraciclib, VEN, AraC, AZA or fadraciclib in combination with VEN, AraC or AZA in physiological growth factor conditions. Annexin V/7aminoactinomycin D (7-AAD) or DAPI (4',6-diamidino2-phenylindole) (BD Biosciences) staining to assess apoptosis by flow cytometry using $1 \times 10^{5}$ cells per condition. Propidium iodide (PI) staining buffer to assess cell cycle progression as per manufacturer's protocol. Fixation/Permeabilization Solution Kit (BD Biosciences) was used prior to MCL-1 or active caspase-3 staining. For staining reagents used, see Supplementary Table S3.

\section{Combination studies}

CompuSyn software (ComboSyn, Inc) was used to investigate the synergism in the cell proliferation assays. The software was based on the Chou-Talalay method for drug combination based on the median-effect equation derived from the mass-action law principle $^{74}$. The $\mathrm{CI}$ provides a quantitative definition for additive effect $(\mathrm{CI}=$ $1)$, synergism $(\mathrm{CI}<1)$ or antagonism $(\mathrm{CI}>1)$ in drug combinations.

\section{Statistics}

Average responses from at least three independent experiments are shown (mean $\pm \mathrm{SD}$ ). Statistical analysis used GraphPad Prism 8 for Student's $t$-test and one-way ANOVA ( $\left." p<0.05,{ }^{* * *} p<0.01,{ }^{* * * *} p<0.001,{ }^{* * * * * * *} p<0.0001\right)$.

\section{Acknowledgements}

The authors thank Alan Hair for processing patient samples, Jennifer Cassels for preparing culture media, Joana Silvestre for creating the heatmap, Chinmay Munje and Caroline Busch for technical support.

\section{Author details \\ 'Paul O'Gorman Leukaemia Research Centre, Institute of Cancer Sciences, College of Medical, Veterinary and Life Sciences, University of Glasgow, Glasgow, UK. ${ }^{2}$ Department of Pathology, Phramongkutklao College of Medicine, Bangkok, Thailand. ${ }^{3}$ Cyclacel Limited, Dundee, UK}

\section{Author contributions}

Contribution: W.C. designed and performed experiments, collected and analyzed data, and wrote the manuscript; Y.-C.H. performed experiments, collected and analyzed data; D.Z. and S.F. provided reagents, discussed experiments and edited the manuscript; H.W. supervised the project, designed experiments, and edited the manuscript; and M.C. supervised the project, designed experiments, and edited the manuscript.

\section{Funding}

This research was funded by a Ph.D. studentship from the Royal Thai Government. Additional funding was provided by Cyclacel Ltd. This study was supported by the Glasgow Experimental Cancer Medicine Center, which is funded by Cancer Research UK (C58789/A251741) and the Chief Scientist's Office, Scotland. Cell-sorting facilities were funded by the Kay Kendall Leukemia Fund (KKL501) and the Howat Foundation.

\section{Conflict of interest}

M.C.: research funding (Cyclacel Ltd, Novartis, BMS, Incyte), advisory board member (BMS, Novartis, Incyte, Pfizer, Daiichi Sankyo), and honoraria (Astellas, BMS, Novartis, Incyte, Pfizer, Takeda, Celgene). D.Z. and S.F. are employees of Cyclacel Ltd. The remaining authors declare no competing interests.

\section{Ethics statement}

Healthy donor and diagnostic primary AML samples were taken in accordance with the Declaration of Helsinki, and Ethics Committee approval (15/WS/0077).

\section{Publisher's note}

Springer Nature remains neutral with regard to jurisdictional claims in published maps and institutional affiliations.

Supplementary information The online version contains supplementary material available at https://doi.org/10.1038/s41420-021-00496-y. 
Received: 10 February 2021 Revised: 4 April 2021 Accepted: 22 April 2021 Published online: 10 June 2021

\section{References}

1. Döhner, H., Weisdorf, D. J. \& Bloomfield, C. D. Acute myeloid leukemia. N. Engl. J. Med 373, 1136-1152 (2015).

2. De Kouchkovsky, I. \& Abdul-Hay, M. 'Acute myeloid leukemia: a comprehensive review and 2016 update'. Blood Cancer J. 6, e441 (2016).

3. Pelcovits, A. \& Niroula, R. Acute myeloid leukemia: a review. R. I. Med. J. (2013) 103, 38-40 (2020).

4. Rangoonwala, H. I., Gootee, J. \& Silberstein, P. T. Epidemiology and survival outcomes in elderly acute myeloid leukemia patients: an NCDB study. Abstracts of the 75th Annual Meeting of the American Society of Hematology. Blood 134, 3840 (2019).

5. Rowe, J. M. The increasing genomic complexity of acute myeloid leukemia. Best. Pr. Res. Clin. Haematol. 27, 209-213 (2014).

6. Hou, H. A. \& Tien, H. F. Genomic landscape in acute myeloid leukemia and its implications in risk classification and targeted therapies. J. Biomed. Sci. 27, 81-94 (2020).

7. Goldman, S. L. et al. Epigenetic modifications in acute myeloid leukemia: prognosis, treatment, and heterogeneity. Front. Genet. 10, 133-148 (2019).

8. Gambacorta, V., Gnani, D., Vago, L. \& Di Micco, R. Epigenetic therapies for acute myeloid leukemia and their immune-related effects. Front. Cell Dev. Biol. 7, 207-226 (2019)

9. Plass, C., Oakes, C., Blum, W. \& Marcucci, G. Epigenetics in acute myeloid leukemia. Semin. Oncol. 35, 378-387 (2008).

10. Akinduro, O. et al. Proliferation dynamics of acute myeloid leukaemia and haematopoietic progenitors competing for bone marrow space. Nat. Commun. 9, 519-531 (2018).

11. Tzoran, I., Rebibo-Sabbah, A., Brenner, B. \& Aharon, A. Disease dynamics in patients with acute myeloid leukemia: new biomarkers. Exp. Hematol. 43, 936-943 (2015).

12. Walter, R. B. et al. Prediction of early death after induction therapy for newly diagnosed acute myeloid leukemia with pretreatment risk scores: a novel paradigm for treatment assignment. J. Clin. Oncol. 29, 4417-4423 (2011).

13. Eleni, L. D., Nicholas, Z. C. \& Alexandros, S. Challenges in treating older patients with acute myeloid leukemia. J. Oncol. 2010, 943823 (2010).

14. Burnett, A. K. et al. A comparison of low-dose cytarabine and hydroxyurea with or without all-trans retinoic acid for acute myeloid leukemia and high-risk myelodysplastic syndrome in patients not considered fit for intensive treatment. Cancer 109, 1114-1124 (2007).

15. Quiroga-Campano, A. L. et al. Personalized and optimized low-dose and intensive chemotherapy treatments for patients with acute myeloid leukemia (AML). Abstracts of the 75th Annual Meeting of the American Society of Hematology. Blood 132, 3500 (2018).

16. Collignon, A. et al. A chemogenomic approach to identify personalized therapy for patients with relapse or refractory acute myeloid leukemia: results of a prospective feasibility study. Blood Cancer J. 10, 64-75 (2020).

17. Illangeswaran, R. S. S., Das, S., Paul, D. Z., Mathews, V. \& Balasubramanian, P. A personalized approach to acute myeloid leukemia therapy: current options. Pharmgenomics Pers. Med. 12, 167-179 (2019).

18. Hourigan, C. S. \& Karp, J. E. Personalized therapy for acute myeloid leukemia. Cancer Discov. 3, 1336-1338 (2013).

19. Stone, R. M. et al. Midostaurin plus chemotherapy for acute myeloid leukemia with a FLT3 Mutation. N. Engl. J. Med. 377, 454-464 (2017).

20. Perl, A. E. et al. Gilteritinib or chemotherapy for relapsed or refractory FLT3mutated AML. N. Engl. J. Med. 381, 1728-1740 (2019).

21. DiNardo, C. D. et al. Durable remissions with ivosidenib in IDH1-mutated relapsed or refractory AML. N. Engl. J. Med. 378, 2386-2398 (2018).

22. Stein, E. M. et al. Enasidenib in mutant $1 \mathrm{DH} 2$ relapsed or refractory acute myeloid leukemia. Blood 130, 722-731 (2017).

23. Stein, E. M. Enasidenib, a targeted inhibitor of mutant $\mathrm{IDH} 2$ proteins for treatment of relapsed or refractory acute myeloid leukemia. Future Oncol. 14, 23-40 (2018).

24. DiNardo, C. D. et al. Venetoclax combined with decitabine or azacitidine in treatment-naive, elderly patients with acute myeloid leukemia. Blood 133, 7-17 (2019).
25. Wei, A. H. et al. Venetoclax combined with low-dose cytarabine for previously untreated patients with acute myeloid leukemia: results from a phase $\mathrm{Ib} / \mathrm{II}$ study. J. Clin. Oncol. 37, 1277-1284 (2019).

26. Zeidner, J. F. et al. Final results of a randomized multicenter phase II study of alvocidib, cytarabine, and mitoxantrone versus cytarabine and daunorubicin $(7+3)$ in newly diagnosed high-risk acute myeloid leukemia (AML). Leuk. Res. 72, 92-95 (2018).

27. Zeidner, J. F. \& Karp, J. E. Clinical activity of alvocidib (flavopiridol) in acute myeloid leukemia. Leuk. Res. 39, 1312-1318 (2015).

28. Zeidner, J. F. et al. Randomized multicenter phase ॥ study of flavopiridol (alvocidib), cytarabine, and mitoxantrone (FLAM) versus cytarabine/daunorubicin $(7+3)$ in newly diagnosed acute myeloid leukemia. Haematologica 100 1172-1179 (2015).

29. Frame, S. et al. Fadraciclib (CYC065), a novel CDK inhibitor, targets key prosurvival and oncogenic pathways in cancer. PLOS ONE 15, e0234103 (2020).

30. Kawakami, M. et al. Next-generation CDK2/9 inhibitors and anaphase catastrophe in lung cancer. J. Natl Cancer Inst. 109, 6-17 (2017).

31. Borthakur, G. M. et al. Combining CDK2/9 Inhibitor CYC065 with Venetoclax, a BCL2 Inhibitor, to Treat Patients with Relapsed or Refractory AML or MDS. Abstracts of the 75th Annual Meeting of the American Society of Hematology. Blood 134, 1379 (2019).

32. Pellerano, M. et al. Targeting conformational activation of CDK2 kinase. Biotechnol. J. 12, 1600531 (2017).

33. Morales, F. \& Giordano, A. Overview of CDK9 as a target in cancer research. Cell Cycle 15, 519-527 (2016).

34. Polier, G. et al. Wogonin and related natural flavones are inhibitors of CDK9 that induce apoptosis in cancer cells by transcriptional suppression of Mcl-1. Cell Death Dis. 2, e182 (2011).

35. Cai, B., Chang, S. H., Becker, E. B. E., Bonni, A. \& Xia, Z. p38 MAP kinase mediates apoptosis through phosphorylation of BimEL at Ser-65. J. Biol. Chem. 281 25215-25222 (2006).

36. Kralova, J., Dvorak, M., Koc, M. \& Kral, V. p38 MAPK plays an essential role in apoptosis induced by photoactivation of a novel ethylene glycol porphyrin derivative. Oncogene 27, 3010-3020 (2008).

37. Deacon, K., Mistry, P., Chernoff, J., Blank, J. L. \& Patel, R. p38 Mitogen-activated protein kinase mediates cell death and p21-activated kinase mediates cell survival during chemotherapeutic drug-induced mitotic arrest. Mol. Biol. Cell 14, 2071-2087 (2003).

38. Eriksson, M. et al. Agonistic targeting of $\mathrm{TLR} 1 / \mathrm{TLR} 2$ induces p38 MAPKdependent apoptosis and NFKB-dependent differentiation of AML cells. Blood Adv. 1, 2046-2057 (2017).

39. Yamaguchi, H., Hsu, J. L. \& Hung, M. C. Regulation of ubiquitination-mediated protein degradation by survival kinases in cancer. Front. Oncol. 2, 15-24 (2012).

40. Hernandez, A. M. et al. Upregulation of p21 activates the intrinsic apoptotic pathway in $\beta$-cells. Am. J. Physiol. Endocrinol. Metab. 304, E1281-E1290 (2013).

41. Foucquier, J. \& Guedj, M. Analysis of drug combinations: current methodological landscape. Pharm. Res. Perspect. 3, e00149 (2015).

42. Do, K. T. et al. Abstract СT037: Phase I safety, pharmacokinetic and pharmacodynamic study of CYC065, a cyclin dependent kinase inhibitor, in patients with advanced cancers (NCT02552953). Abstracts of the AACR 109th Annual Meeting. Cancer Res. 78, CT037 (2018).

43. Teh, T. C. et al. Enhancing venetoclax activity in acute myeloid leukemia by cotargeting MCL1. Leukemia 32, 303-312 (2018).

44. Leonard, S. M., Perry, T., Woodman, C. B. \& Kearns, P. Sequential treatment with cytarabine and decitabine has an increased anti-leukemia effect compared to cytarabine alone in xenograft models of childhood acute myeloid leukemia. PLOS ONE 9, e87475 (2014).

45. Adachi, Y., Ishikawa, Y. \& Kiyoi, H. Identification of volasertib-resistant mechanism and evaluation of combination effects with volasertib and other agents on acute myeloid leukemia. Oncotarget 8, 78452-78465 (2017).

46. Min, C. et al. Selective inhibitors of histone deacetylases 1 and 2 synergize with azacitidine in acute myeloid leukemia. PLoS ONE 12, e0169128 (2017).

47. Anderson, M. A. et al. The BCL2 selective inhibitor venetoclax induces rapid onset apoptosis of CLL cells in patients via a TP53-independent mechanism. Blood 127, 3215-3224 (2016).

48. Heasman, S. A., Zaitseva, L., Bowles, K. M., Rushworth, S. A. \& MacEwan, D. J. Protection of acute myeloid leukaemia cells from apoptosis induced by frontline chemotherapeutics is mediated by haem oxygenase-1. Oncotarget 2, 658-668 (2011). 
49. Hollenbach, P. W. et al. A comparison of azacitidine and decitabine activities in acute myeloid leukemia cell lines. PLoS ONE 5, e9001 (2010).

50. Döhner, H. et al. Diagnosis and management of AML in adults: 2017 ELN recommendations from an international expert panel. Blood 129, 424-447 (2017).

51. Tetsu, O. \& McCormick, F. Proliferation of cancer cells despite CDK2 inhibition. Cancer Cell 3, 233-245 (2003).

52. Chytil, A. et al. Construction of a cyclin D1-Cdk2 fusion protein to model the biological functions of cyclin D1-Cdk2 complexes. J. Biol. Chem. 279, 47688-47698 (2004)

53. Santo, L., Siu, K. T. \& Raje, N. Targeting cyclin-dependent kinases and cell cycle progression in human cancers. Semin. Oncol. 42, 788-800 (2015).

54. Bacon, C. W. \& D'Orso, I. CDK9: a signaling hub for transcriptional control. Transcription 10, 57-75 (2019).

55. Nelson, D. M. et al. Flavopiridol induces BCL-2 expression and represses oncogenic transcription factors in leukemic blasts from adults with refractory acute myeloid leukemia. Leuk. Lymphoma 52, 1999-2006 (2011).

56. Glaser, S. P. et al. Anti-apoptotic Mcl-1 is essential for the development and sustained growth of acute myeloid leukemia. Genes Dev. 26, 120-125 (2012)

57. Hermanson, D. L., Das, S. G., Li, Y. \& Xing, C. Overexpression of Mcl-1 confers multidrug resistance, whereas topoisomerase $\| \beta$ downregulation introduces mitoxantrone-specific drug resistance in acute myeloid leukemia. Mol. Pharm. 84, 236-243 (2013).

58. Kavela, S. et al. PNUTS functions as a proto-oncogene by sequestering PTEN. Cancer Res. 73, 205-214 (2013).

59. Karp, J. E. et al. Phase I and pharmacokinetic study of flavopiridol followed by 1-beta-D-arabinofuranosylcytosine and mitoxantrone in relapsed and refractory adult acute leukemias. Clin. Cancer Res. 11, 8403-8412 (2005).

60. Hao, S., Chen, C. \& Cheng, T. Cell cycle regulation of hematopoietic stem or progenitor cells. Int. J. Hematol. 103, 487-497 (2016).

61. Malumbres, M. \& Barbacid, M. Cell cycle, CDKs and cancer: a changing paradigm. Nat. Rev. Cancer 9, 153-166 (2009).

62. Richard-Carpentier, G. \& DiNardo, C. D. Single-agent and combination biologics in acute myeloid leukemia. Hematol. Am. Soc. Hematol. Educ. Program 2019, 548-556 (2019).

63. He, N. et al. Human polymerase-associated factor complex (PAFc) connects the super elongation complex (SEC) to RNA polymerase II on chromatin. Proc. Natl Acad. Sci. USA 108, E636-E645 (2011).
64. Scholz, A. et al. Abstract 3022: BAY 1143572, a first-in-class, highly selective, potent and orally available inhibitor of PTEFb/CDK9 currently in Phase I, shows convincing antitumor activity in preclinical models of acute myeloid leukemia (AML). Abstracts of the AACR 107th Annual Meeting. Cancer Res. 76, 3022 (2016).

65. Kuo, F. C., Mar, B. G., Lindsley, R. C. \& Lindeman, N. I. The relative utilities of genome-wide, gene panel, and individual gene sequencing in clinical practice. Blood 130, 433-439 (2017).

66. Estey, E. H. Acute myeloid leukemia: 2019 update on risk-stratification and management. Am. J. Hematol. 93, 1267-1291 (2018).

67. Bertoli, S. et al. Time from diagnosis to intensive chemotherapy initiation does not adversely impact the outcome of patients with acute myeloid leukemia. Blood 121, 2618-2626 (2013).

68. Moujalled, D. M. et al. Combining BH3-mimetics to target both BCL-2 and MCL1 has potent activity in pre-clinical models of acute myeloid leukemia. Leukemia 33, 905-917 (2019).

69. Phillips, D. C. et al. A novel CDK9 inhibitor increases the efficacy of venetoclax (ABT-199) in multiple models of hematologic malignancies. Leukemia 34, 1646-1657 (2019)

70. Chen, R. et al. Abstract 3905: Strategic combination of the cyclin-dependent kinase inhibitor CYC065 with venetoclax to target anti-apoptotic proteins in chronic lymphocytic leukemia. Abstracts of the AACR 109th Annual Meeting. Cancer Res. 78, 3905 (2018).

71. Pepper, C. et al. Mcl-1 expression has in vitro and in vivo significance in chronic lymphocytic leukemia and is associated with other poor prognostic markers. Blood 112, 3807-3817 (2008).

72. Kim, W. et al. The CDK9 inhibitor, alvocidib, potentiates the non-clinical activity of azacytidine or decitabine in an MCL-1-dependent fashion, supporting clinical exploration of a decitabine and alvocidib combination. Abstracts of the 74th Annual Meeting of the American Society of Hematology. Blood 132, 4355 (2018).

73. Rao, X., Huang, X., Zhou, Z. \& Lin, X. An improvement of the $2(-\Delta \Delta C T)$ method for quantitative real-time polymerase chain reaction data analysis. Biostat. Bioinforma. Biomath. 3, 71-85 (2013).

74. Chao T. C. \& Martin N. CompuSyn for Drug Combinations: PC Software and User's Guide: A Computer Program for Quantitation of Synergism and Antagonism in Drug Combinations, and the Determination of IC50 and ED50 and LD50 Values (ComboSyn Inc, 2005). 